\title{
The Application of Blockchain in Higher Education Field
}

\author{
Wenqing ZHAO \\ School of Control and Computer Engineering \\ North China Electric Power University \\ Baoding 071003, China
}

\author{
Zhuoying MA \\ School of Control and Computer Engineering \\ North China Electric Power University \\ Baoding 071003, China
}

\begin{abstract}
The blockchain technology is seen as revolutionary technology, which obtains attention from various fields in many countries. In China, students information in higher education needs to be managed by the third part-China Credentials Verification. Although this website brings a lot of convenience to students, it still exis ts some dis advantages. Through analyzing the application of blockchain technology in finance field, the key feature - decentralized can also be effectively applied in the education field to improve the work efficiency of verifying degree, storing considerable data or transmitting students' information in the way of point-to-point and so on. Through exploring the modern of 'blockchain+education', considerable students' information in database can be stored in the blockchain and can be traced by companies to conveniently query their records. This paper will discuss the application landscape of blockchain te chnology in higher education in new view.
\end{abstract}

Keywords-Blockchain Technology; Education Application; 'Blockchain+education' Modern

\section{INTRODUCTION}

As the bottom technology of bitcoin, blockchain technology firstly attracts much attention from financial industry. Blockchain technology is a kind of data structure, which links the block together and realizes data transmission in the way of decentralization without the third part participation. Its principle technologies, such as decentralization, distributed accounting technology and intelligent contract ,not only are widely applied in the field of finance, electric data, information safety and so on ,but also have wonderful values in the higher education field and it can promote education field informational and intelligent.

In overseas, the research on blockchain technology started earlier than China. For example, the England government's report-distributed ledger: Blockchain Technology points that blockchain technology is able to provide a new credit mechanism to many kinds of services[6]. The America governments also adopted a serious of plans-'blockchain advocation activity'.

Compared with overseas, the development of blockchain technology in China is at the initial stage the higher education students information management works became informational began 1990s, along with the development of information technology, its management modern transformed from signal computer to the Internet. But many educational institutions play as an education master to monopolize

Supported by double first-class graduate construction project of North China Electric Power University learning certification, so learners, teachers and learning partners hardly have autonomy to the process and results of learning[1] . For example, in the aspect of diploma and degree application, the China Credentials Verification website supplies the service of certifying them. Although this website exerts some good effect on certification online in some informal occasion, paper certifications often received more recognition in school admission or work employment.

Based on some exist on students information management in higher education filed ,this thesis will focus on exploring a new education status under the emerging blockchain technology referring to the development experience in financial industry and others.

\section{THE DEFINITION AND THE KEY TECHNOLOGY}

\section{A. The Definition of Blockchain}

Blockchain consists block with chain: block - it is a data block which records the all cryptography information within some range of time; chain - it describes the relationship between the block and the next, so they constitute the blockchain. Attributing to each block includes the all trading information data, which don't need the third part to supply service, so each block is equal to other blocks. In a narrow sense, blockchain is a chained data structure which associates data blocks together according to time order[2]. In essence, blockchain is a distributed database which needs all the participants to work together.

\section{B. The Key Technology of Blockchain Technology}

- Distributed Ledger Technology: Blockchain technology is also called distributed ledger technology. Now the internet needs a central server to connect each node. If the central server doesn't work, the cloud database will also stop updating data. Rather, the blockchain technology doesn't rely on the central service. So works are finished jointly by different nodes which distribute in different districts and can record the corresponding information. Distributed ledger is a database that the networks participants can share, copy and synchronize data or can record the mutual trade data. And using the following technologies: public key, private key and digital sign to realize data storing become safe and accurate. It makes each node wherever in any locations or networks can share 
information conveniently. So the technology effectively decreases time cost.

- Asymmetric Encryption Algorithm: Blockchain technology uses the asymmetric encryption to encrypt data. Work proof and consensus mechanism are used to resist outside attacks and assure the data unmodified. The algorithm includes two keys: public key and private key. They phase match. If using the public key to encrypt data, the private key is used to deciphering and vice versa. This algorithm is safer than symmetric encryption algorithm.

- Intelligent Contract: It is a computer progress developed by the cryptography and other subjects. It is made by the all participants. In the contract, programmers use codes which include executed conditions to specify users' rights and obligations. Once the coding work is finished, the contract will upload data to the internet. And it will termly check relative incidents and trigger conditions. If some nodes are verified, which will firstly sign the incident to verify the last step. The successfully executed contracts will be eliminated from blocks and others will continue to waiting the next trading. This key technology has many merits: firstly, ensuring information open, transparent, unmodified and avoiding effectively that cheating events occur. Secondly, controlling the blockchain asset and storing or transforming digital currency or learning data in each node. So the trade information can be traced and stored permanently. Thirdly, intelligent contract program is executed automatically by blockchain, so people can not intervene or modify data. In one side, it can improve the efficiency of platform trading and can meet the consumers' requires to get knowledge. In other side, it can assure platforms stable. Lastly, intelligent contract don't need the third part. It can realize that between learners and train institutions, learners and teachers and different institutions trade point-to-point. Not only it can save the fee of operating and maintaining platforms, but also can provide online learning service.

\section{The features of blockchain technology}

Blockchain technology is proposed by the thesis: bitcoinsa kind of point-to-point digital crash system published in 2008. This thesis pointed out that the blockchain technology is a new distributed basic frame under the point-to-point networks, which uses the chain data structure to verify and store massive data, uses distributed node consensus algorithm to produce and update data, uses cryptography to assure the safety of data transmission and access, uses the intelligent contract to program and operate data. The following sections will overview the features of blockchain technology at length.

- Decentralized: Now we live in a centralized society. For example, we need to rely on the internet platform to purchase one thing, so the website plays the role as a intermediary institution to manage buyers capital. This way is complex and tedious. However, with the appearance of blockchain technology it will not need this centralized system modern. Blockchain technology utilizes the point-to-point internet connection modern to trade and each user is a node, so trades can be finished directly by users without the third part participation.

- Traceable: Supported by blockchain technology, the internet can do marks referring to $\mathrm{IP}$ addresses when each nodes or users correspond to other users. And using chain storing structure including time stamp to store data on the block, so the technology increases time dimensions and it is able to trace the source of data[4].

- Highly trusted: The decentralized modern realizes the nodes interactive directly. But the mutual should create a trust mechanism to assure data safety. On the basic of consensus mechanism, the mutual don't need to obtain trust from each other and it can be achieved that the data can't be modified. The data on the blockchain can supply the permanent backups, which are public and transparent.

- Anonymous: Technologically, each block doesn't need to verify their identity information. So information transmits anonymously. This feature is achieved by the consensus mechanism. Under this feature, not only can meet users' requires, but also can increase the sense of mutual trust.

\section{THE OVERVIEW OF THE APPLICATION OF BLOCKCHAIN TECHNOLOGY IN HIGHER EDUCATION FIELD}

\section{A. The discussion about blockchain technology application modern in higher education field}

In 2016, the Ministry of Industry and Information Technology issued Chinese blockchain technology and application white paper which pointed out that the transparent and unmodified natures of blockchain technology can apply entirely to the education field. Now no matter in China or overseas, students' information management works both have the following flaws. Firstly, people who need to verify the diploma or degree should pay some money to platforms. Secondly, both verified institutions and schools need to spend much time to verify the realness about them. Thirdly, schools' database records considerable information. So the cost of maintaining those data is expensive. Lastly, the verified work is often based on the workers' subjective judgment, so it exists unforeseeable credit risks. The emergence of blockchain technology can address the above problems.

\section{1) Enhance the intellectual property protect}

The traceability about digital currency can effectively avoid the appearance of illegal events such as tax evasion, money laundering. Likewise, the feature can be greatly used in higher education to deal with the questions about intelligent property protection.

The key function about blockchain technology is the truth mechanism which using encryption and consensus algorithm to assure data can't be modified and fabricated. So using this technology can contribute to creating safe and credible digital economic rules and regulars. In one side, using distributed 
ledger can record the information about students' school roll, school file, diploma and award-winning distributed in different districts or schools to conveniently track the students' information in higher school and to create good credit ecology. When students apply for jobs, companies can comprehensively know students' records and academic abilities. The distributed storing is favorable to the information's junction between companies and schools[3]. In the other side, using blockchain technology can digitally prove academic achievements, which provide authoritative proof to avoid academic dissension. At the same time, the digital proof attaching the unique time stamp can radically assure data intact and coherent.

\section{2) Establish a credible and convenient certifications} verification system

Some education institutions have try to record the learning certification on the blockchain to provide convenient and credible certifications. According to a report, an app which is similar to 'bitcoin wallet' can be carried in anywhere and can be showed at any time. It reclaims the students' ownership to the learners. Although the learners can not modify the contents of records, they can query and show data to the demand sides like companies. To avoid the records are modified deliberately, learners can provide private key to the demand side.

\section{3) Realize considerable data storing}

Now the data in the networks about student' information is massive. Those data needs to be maintained by center servers, so the cost of maintaining and optimizing data is expensive. But the decentralized features of blockchain technology can be used to address the problem. The data can be stored orderly in the blockchain and data will be attached with time stamp to help educational workers query information at any time. And not only the administer can query the data, but also each participants can do.

\section{4) Realize resources share}

Based on blockchain technology, the digital economic system is a point-to-point system which opens to the all participants. The point-to-point feature can realize economic cooperation between different institutions and organizations. In higher education field, using it can also share all kinds of excellent education resource to learners- the excellent courses, books and literature. So the learners can conveniently obtain educational resources. The data sharing between different organizations can further promote the ability of public service platforms in education and can gradually realize open between resource and management platform. Meanwhile, utilizing the technology of blockchain can supervise the behaviors of data abuse or leakage [6].

\section{5) Create a positive study atmosphere}

One key technology-intelligent contract can do generous verified and managed works automatically in financial field. In higher education, using it can also realize learning resources uploaded, verified and shared automatically. Otherwise, it can create a positive study atmosphere through automatically supervising the atmosphere in learning community and eliminating some bad discussions.

\section{B. The meaning of 'blockchain +education' modern}

In the traditional education field, no matter the face-to-face teaching or studying online are restricted by the schools' management. In some extents, although the certification issued by schools can show students' achievement , students are responsible to learning information and should protect seriously all kinds of learning feedback which jobs or further study need. However, recording and storing concerned information occupied learners much time and energy. For example, the admission managers in education institutions lack relative ways and resources to verify the accuracy of data. But applying distributed and open learning management platforms can effectively deal with the above-mentioned questions. Specifically, the meaning of 'blockchain +education' can reflect on the following respects.

Firstly, the learners and teachers will be the managers of teaching resources and information. Secondly, to some people who can not finish traditional education, the blockchain technology can combine the scores or study results from different organizations together. And through applying the certification from education institutions to get concerned degree certifications. Now to adapt the learning certification based on the blockchain technology, the England open university's Knowledge Media Institute have issued combined 'Micro-credentials' -a innovating technology. Thirdly, to some low incomes people or countries, using the digital currency can increase invest in education field. According to the World Bank statistics, the total amount of remit money to the developing country is more than $\$ 4310$ billion, but the bank need to charge $20 \%$ benefits according to different districts. If using the intelligent contract and digital currency based on the blockchain technology to trade point-topoint, the value of remit money will improve to $10 \%$ or even $20 \%$. So in some extents, this can increase the expenditure in the education field[1].

\section{The challenge of 'blockchain +education'}

Firstly, each country has different attitudes to the blockchain technology, so it inevitably restricts the development of blockchain. Although the decentralized feature of blockchain convenient users, in some extent, it reduces the power of management institution and impacts the laws enforcement[4].

Secondly, although the blockchain technology has applied in financial field, some unsafe events such as currency lose or steal occasionally occur. So in the higher education field, we don't know that the similar phenomenon whether will occur in one day.

Lastly, only a small number of educational workers can master the blockchain technology. In order to realize that this technology is used widely, the leaders should know the importance in mind ,the managers should assist leaders and the teachers should respond positively and propagate greatly.

\section{CONCLUSION}

In present, as far as the blockchain technology whether to apply it is not the doubt affects, but how to apply this technology is the key matter. For example, the universities use 
the new platform based on the blockchain technology to meet learners needs which students can choose courses or join learning activities from different institutions. So it radically increases learning chances. Now a large number of educational organizations are using this technology to explore an emerging education platform. So the 'blockchain +education' modern will be getting greatly developed in the future.

This thesis is divided into four major sections and stresses the blockchain technology application in higher education. Beyond all doubts, this technology will have massive application potential in the higher education aspect. In conclusion, the 'blockchain +education' modern can be applied in the following aspects: firstly, storing and recording learning contents and scores; secondly, verifying school diploma or degree; thirdly, managing automatically about student information. Certainly, in the future it will face the following challenges: firstly, the corresponding speed of the blockchain should be improved; secondly, the storing space needs to be expanded; thirdly, it is difficult to assure that the clients' information is leaked by others. Hoping that we can further explore some education application cases in the future to reform the higher education modern.

\section{REFERENCES}

[1] Tao Xu.The Research about the Status and Application Value of 'Blockchain Technology+' Education[J].DISTANCE LEARNING MAGAZINE, 2017, 35(02):19-28. (In Chinese)

[2] Qing Li, Xin Zhan. Blockchain: Based on Technology to Push Education Field Open and Credible[J].DISTANCE LEARNING MAGAZINE, 2017, 35(01):36-44. (In Chinese)

[3] Xianmin Yang, Xin Li, Huanqing Wu and Keyun Zhao. The Application Modern and Challenge about Blockchain Technology in Education Field [J].Modern DISTA NCE LEA RNING RESEARCH, 2017(02):34-45. (In Chinese)

[4] Feng Man, Chen Lin. Blockchain. technology: A Emerging Technology Pushing Education Reform[J].Chinese Medical Education Technology, 2017, 31(06):613-617. (In Chinese)

[5] Guanghua Qia, Kongqing Zhu, Dezhong Zhao and Shijie Wang.The Research of Diploma and Degree Verified Modern under The Blockchain Technology[J].OPERATION AND MANAGEMENT, 2018(03):142-146. (In Chinese)

[6] Hua Du. The Value Reconstruction and Path Innovation of The Development of Higher Education by Blockchain Technology[J]. MODERN EDUCATION TECHNOLOGY, 2017, 27(10):55-60. (In Chinese) 